\title{
Possible companions in low-mass eclipsing binaries: V380 Dra, BX Tri, and V642 Vir
}

\author{
M. Wolf ${ }^{1}$, H. Kučáková ${ }^{1,2,3,4}$, P. Zasche ${ }^{1}$, L. Šmelcer ${ }^{4,5}$, K. Hornoch ${ }^{2}$, \\ K. Hoňková ${ }^{4}$, J. Juryšek ${ }^{4}$, M. Mašek ${ }^{4,6}$ and M. Lehký ${ }^{4}$ \\ ${ }^{1}$ Astronomical Institute, Faculty of Mathematics and Physics, Charles \\ University, Praha 8, Czech Republic, (E-mail: wolf@cesnet.cz) \\ 2 Astronomical Institute, Academy of Sciences of the Czech Republic, \\ Ond??ejov, Czech Republic \\ 3 Institute of Physics, Faculty of Philosophy and Science, Silesian University \\ in Opava, Czech Republic \\ 4 Variable Star and Exoplanet Section of the Czech Astronomical Society \\ 5 Observatory Valašské Meziřı́čí, Czech Republic \\ ${ }^{6}$ Institute of Physics, Czech Academy of Sciences, Praha 8, Czech Republic
}

Received: November 1, 2019; Accepted: December 1, 2019

\begin{abstract}
We present the new results of our long-term observational project to analyze the orbital period variations of low-mass eclipsing binaries. More then 200 new precise mid-eclipse times recorded with a CCD were obtained for three eclipsing binaries with short orbital periods: V380 Dra $(P=0.49)$, BX Tri (0.19), and V642 Vir (0.52). Observed-minus-calculated diagrams of the stars were analyzed using all reliable timings, and new parameters of the light-time effect were obtained. We derived for the first time the short orbital periods of possible third bodies of 10-20 years for these objects. We calculated that the minimum masses of the third component are close to $0.2 \mathrm{M}_{\odot}$, which corresponds to the mass of M4 - M5 red dwarfs. The multiplicity of these systems also plays an important role in the precise determination of their physical parameters.
\end{abstract}

Key words: binaries: eclipsing - stars: late-type - stars: fundamental parameters - stars: individual (V380 Dra, BX Tri, V642 Vir)

\section{Introduction}

Low-mass binaries (LMBs) and their multiple systems play an important role in stellar astrophysics. Their origin and evolution is still an unresolved question in the star formation theory. Moreover, observations of low-mass stars show a discrepancy between estimated and modeled parameters, where the models give some $10 \%$ smaller radii than observations while their effective temperatures are some 5\% higher (e.g. Ribas et al. 2008). Our previous period studies of similar eclipsing LMBs presented in Wolf et al. $(2016,2018)$ revealed several triple 
Table 1. The LITE parameters of three selected LMBs: orbital and third body periods (in days and years), amplitudes, eccentricities, expected masses of binary components, minimal masses of the possible third body, and numbers of primary and secondary mid-eclipse times used in our calculation.

\begin{tabular}{lccc}
\hline \hline System & V380 Dra $=$ & BX Tri $=$ & V642 Vir $=$ \\
& NSVS 1178845 & NSVS 6550671 & NSVS 10441882 \\
\hline$T_{0}$ [HJD-2400000] & 54272.4586 & 51352.0695 & 51274.6257 \\
Period [day] & 0.493736 & 0.192635 & 0.516644 \\
$P_{3}$ [day] & 5595 & 3161 & 7405 \\
$P_{3}$ [year] & 15.5 & 8.65 & 20.3 \\
Amplitude [day] & 0.0041 & 0.00465 & 0.00487 \\
Eccentricity & 0.34 & 0.55 & 0.31 \\
Omega [deg] & 202 & 330 & 61 \\
Time of periastron & 52020 & 55285 & 51640 \\
$M_{1}+M_{2}[M \odot]$ & $0.77+0.62$ & $0.51+0.26$ & $0.67+0.63$ \\
$M_{3, \text { min }}[M \odot]$ & 0.163 & 0.216 & 0.147 \\
$K[\mathrm{~km} / \mathrm{s}]$ & 1.55 & 3.8 & 1.32 \\
$N_{\text {pri }}+N_{\text {sec }}$ & $45+34$ & $320+75$ & $38+32$ \\
$\Sigma w(O-C)^{2}$ & 0.00023 & 0.00059 & 0.00008 \\
\hline \hline
\end{tabular}

systems (e.g. GU Boo and YY Gem). Here we report on a long-term mid-eclipse times campaign of three more selected LMBs. These systems are all relatively well-known low-mass binaries with short orbital periods up to 12 hours.

\section{Observations}

The systematical long-term CCD eclipse and light-curve monitoring of many eclipsing LMBs has been performed since 2006. These relatively faint objects with a short orbital periods, and rapid and deep eclipses, are good targets for practical exercises in photometry. The 0.65-m Mayer reflecting telescope (D65) in Ondřejov observatory, Czech Republic, CCD camera G2-3200, VRI filters, and remote access was used. Precise photometry was also carried out at the Valašské Meziříčí observatory using the 0.3-m Celestron Ultima telescope, and with the $0.4 \mathrm{~m}$ Jan Šindel Telescope operated in the dome of the Astronomical Society in Hradec Králové. The Bootes-2 telescope ${ }^{1}$ in Spain (0.6-m RC telescope and CCD camera Andor iXon, Jelinek et al. 2016) was also used during several nights. A standard calibration (dark frame, flat field) was applied to the observed CCD frames. Aрнот, an aperture photometry and astrometry software, was used at Ondřejov observatory. C-MUNIPACK ${ }^{2}$ was used to reduce our CCD time series obtained at Valašské Meziříčí and Hradec Králové. Differential

\footnotetext{
${ }^{1}$ http://bootes.iaa.es/

${ }^{2}$ http://c-munipack.source.forge.net/
} 


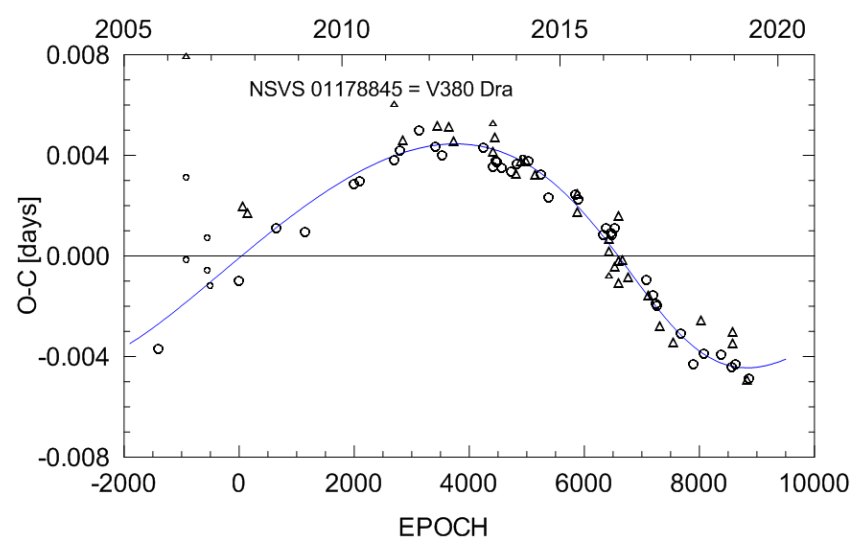

Figure 1. The current O-C diagram of the eclipsing binary V380 Dra. Primary and secondary eclipses are plotted as circles and triangles, respectively. Larger symbols correspond to more precise CCD measurements. The sinusoidal curve represents our solution of the LITE with the period of $15.5 \mathrm{yr}$ and the amplitude of 6 min.

photometry was performed using suitable comparison stars. All new times of primary and secondary minima and their errors were generally determined by fitting the light curve by Gaussians or polynomials of the third or fourth order; we used the least squares method.

\section{Period variation and light-time effect}

The period analysis of three selected LMBs was performed using all available mid-eclipse times found in the literature as well as our newly measured times. One of the best method to detect the third body orbiting the eclipsing binary is the light travel delay, or so-called light-time effect (LITE), associated with orbital motion of the third body (e.g. Mayer 1990). The precise mid-eclipse time estimation enabled us to find small apparent period changes and derive the minimum mass of the body on the wider orbit. Seven independent variables were determined in this procedure: the orbital period of the binary, the orbital period of the third body, the semiamplitude of LITE, the eccentricity of the outer orbit, the periastron passage time of the third body, the zero epoch of the eclipsing binary, and finally the corresponding position of the periastron. All computed LITE parameters are given in Table 1, and the corresponding O-C diagrams are shown in Figs. 1, 2, and 3. 


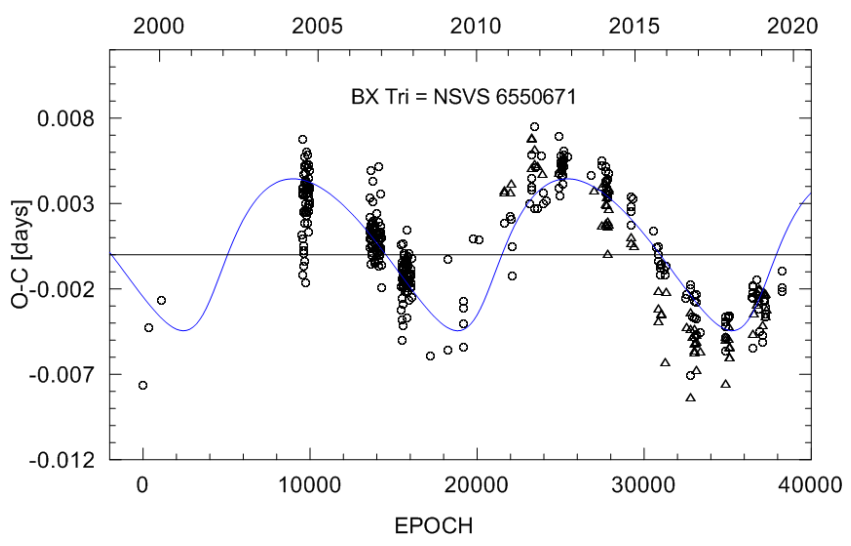

Figure 2. Cyclic period changes in the current O-C diagram of BX Tri. See legend to Fig. 1. The curve represents our solution of LITE with the short period of $8.65 \mathrm{yr}$.

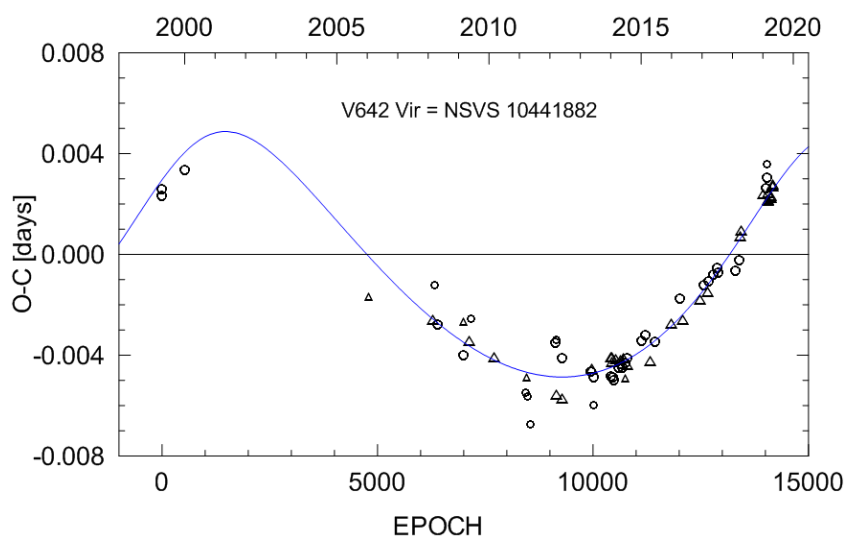

Figure 3. The O-C diagram of V642 Vir. See legend to Fig. 1. The sinusoidal curve represents the LITE with the period of $20.3 \mathrm{yr}$ and an amplitude of $7 \mathrm{~min}$.

\section{Conclusions}

Our study provides accurate information on period changes of three mainsequence LMBs. The LITE period has been presented here for the first time. For V380 Dra and V642 Vir the third-body orbital period is not covered satisfactorily, so these results must be taken as preliminary. The minimal masses of possible third bodies correspond to red-dwarf stars of spectral types M4-M5 (Pecaut \& Mamajek 2013) ${ }^{3}$ with significant third light. The sample of well-known LMBs

${ }^{3}$ http://www.pas.rochester.edu/ emamajek/EEM_dwarf_UBVIJHK_colors_Teff.txt 
needs to be increased, so observations of additional systems would be very useful. It would be also desirable to obtain new spectroscopic observations.

To date about 20 LMBs and hundreds of their light curves are displayed in our database. These objects were selected originally from Shaw \& LópezMorales (2007). The interesting post-common-envelope binaries (HW Vir, NY Vir) and well-known cataclysmic variables (HT Cas, EX Dra, DQ Her, RW Tri, TT Tri, DW UMa) are also included. All objects mentioned above are open to future collaboration. The multicolor photometry of individual systems and the list of precise mid-eclipse times are available on request.

Acknowledgements. This research is part of an ongoing collaboration between professional astronomers and the Czech Astronomical Society, Variable Star and Exoplanet Section. The research of MW and PZ was supported by the Charles University grant Progress Q47. KH and HK were supported by the project RVO: 67985815. The authors would also like to thank Lenka Kotková, Ondřejov, and Jan Vraštil, Charles University, for their important contribution to photometric observations. The following internet-based resources were used in research for this paper: the SIMBAD database operated at CDS, Strasbourg, France, the NASA's Astrophysics Data System Bibliographic Services, and the O-C Gateway of the Czech Astronomical Society. ${ }^{4}$

\section{References}

Jelinek, M., Castro-Tirado, A.J., Cunniffe, R., et al.: 2016, Adv. Astron., 1928465

Mayer, P.: 1990, Bull. Astr. Inst. Czech. 41, 231

Pecaut, M.J. \& Mamajek, E.E.: 2013, ApJS 208, 9

Ribas, I., Morales, J.C., Jordi, C. et al.: 2008, Mem. Soc. Astr. It. 79, 562

Shaw, J.S. \& López-Morales, M.: 2007, ASP Conference Series 362, 15

Wolf, M., Zasche, P., Kučáková, H. et al.: 2016, A\&A 587, 82

Wolf, M., Kučáková, H., Zasche, P. et al.: 2018, A\& A 620, A72

\footnotetext{
${ }^{4}$ http://var.astro.cz/ocgate/
} 